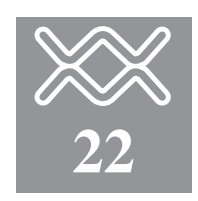

\title{
Il posizionamento del catetere venoso centrale: dove e come
}

\author{
P.A. Conz \\ U.O.C. Nefrologia e Dialisi \\ Ospedale Vittorio Emanuele III, Monselice (PD)
}

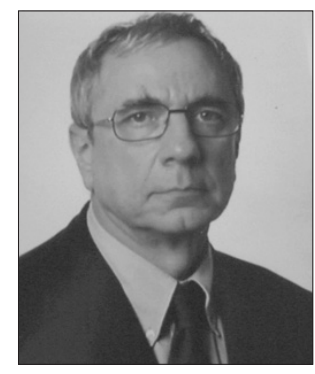

Ta fistola artero-venosa sottocutanea di Cimino-Brescia è stata introdotta nella pratica clinica nel 1966 e a tutt'oggi rimane l'accesso vascolare preferenziale per l'emodialisi a lungo termine.

Orbene, se l'accesso vascolare e la fistola artero-venosa nativa in particolare rappresentano il presupposto essenziale per l'esecuzione del trattamento dialitico extracorporeo, esistono delle condizioni che impongono il ricorso a un accesso vascolare di emergenza che trova la sua espressione nel catetere venoso centrale (CVC).

Questa evenienza si verifica più frequentemente quando, in caso di insufficienza renale acuta non ostruttiva, il trattamento dialitico extracorporeo assume carattere di urgenza.

Il ricorso a un CVC diventa necessario nel paziente uremico in trattamento dialitico regolare qualora sopraggiunga una trombosi acuta del preesistente accesso vascolare o qualora lo stesso sia temporaneamente inutilizzabile (ematoma, processi flebitici ecc.). Va tuttavia ricordato che un accesso vascolare di emergenza, qualunque esso sia (accesso giugulare o succlavio o femorale), deve rispettare alcuni requisiti fondamentali:

1) il suo posizionamento deve essere di relativa, facile attuazione;

2) gli effetti collaterali devono essere $i$ minori possibili;

3) la sua utilizzazione deve essere possibile subito dopo la sua realizzazione;

4) il flusso ematico deve essere sufficiente a garantire il trattamento dialitico.

\section{Tecnica manuale di cannulazione di vena centrale}

La tecnica di Seldinger descritta la prima volta nel 1953 (1) e che prevede lo localizzazione manuale del punto di inserzione dell'ago guida nel vaso da cannulare, è quella che ancora oggi è maggiormente utilizzata da chi (soprattutto intensivisti e rianimatori) pratica la cannulazione di vena centrale. La tecnica può essere schematizzata nei seguenti momenti operativi:

1) Introduzione nel vaso da cateterizzare di un ago montato su siringa.

2) Rimozione della siringa e introduzione del filo guida metallico con punta flessibile o a $\mathrm{J}$ attraverso l'ago inserito nel vaso.

3) Rimozione dell'ago lasciando la guida nel vaso.
4) Esecuzione di piccola incisione con la punta del bisturi nel punto di emergenza cutanea della guida.

5) Inserimento del dilatatore nel vaso attraverso il filo guida metallico.

6) Rimozione del dilatatore.

7) Inserimento del catetere nel vaso attraverso il filo guida.

8) Rimozione del filo guida e ancoraggio del catetere sulla cute mediante punto di sutura.

La vena giugulare interna destra per il trattamento dialitico extracorporeo è generalmente riconosciuta rappresentare la scelta principale per il posizionamento del CVC (2); tale scelta è giustificata principalmente da tre ordini di fattori. Infatti, la VGI dx permette:

1) una corsa relativamente diretta del catetere verso la vena cava superiore;

2) una più bassa incidenza di stenosi in confronto all'accesso vascolare succlavia;

3) non causa o sostiene un significativo aumento della pressione intracranica.

Il cateterismo della vena giugulare interna è da preferirsi al cateterismo della vena succlavia perché gravato da minore incidenza di complicanze. La vena giugulare interna, previa anestesia locale, viene punta a livello del 


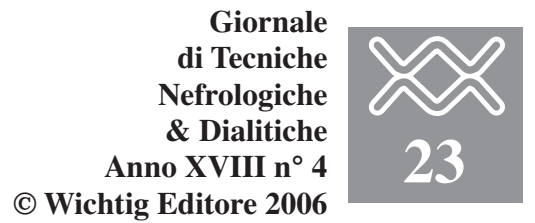

triangolo di Sedillot nel punto di intersezione dei fasci lunghi del muscolo sternocleidomastoideo.

Sono per lo più utilizzate cannule a doppio lume, della lunghezza variabile da 15 a $20 \mathrm{~cm}$ in poliuretano. Si utilizzano anche cateteri a due vie in silicone radiopaco, di lunghezza variabile da 25 a $40 \mathrm{~cm}$ che vengono inseriti sia per via percutanea sia chirurgicamente, e posizionati attraverso un tunnel sottocutaneo. La tecnica chirurgica è abbastanza complessa e negli ultimi anni è stata progressivamente sostituita dalla tecnica percutanea.

La possibilità di permanenza in situ del catetere è ottima e si aggira intorno alle quattro settimane; sono stati comunque segnalati casi di permanenza del catetere per tempi anche superiori ai sei mesi. Il flusso ematico è per lo più superiore ai $200 \mathrm{ml} /$ minuto, la pressione venosa media di rientro si aggira sui $100-150 \mathrm{mmHg}$ e il ricircolo viene calcolato intorno al $10-25 \%$. Le complicazioni più frequenti del cateterismo della giugulare sono elencate nella Tabella I.

È ampiamente riconosciuto che l'infezione rappresenta la complicazione più frequente e che essa nella maggior parte dei casi si associa alla condizione di stenosi-trombosi. Nel caso di infezione si deve rimuovere il catetere ed è buona regola eseguire l'esame colturale della punta del catetere stesso. Non infrequente è la comparsa di ematoma laterocervicale per puntura accidentale della carotide esterna: la pronta rimozione dell'ago e una corretta manovra compressiva per $10-15$, permettono di arrestare il sanguinamento limitando così lo stravaso ematico: una volta accertato l'arresto del sanguinamento è possibile ripetere la manovra di incannulamento dalla parte opposta.

Aritmie ventricolari possono insorgere durante la manovra di incannulamento se la punta del catetere scende fino in cavità ventricolare destra $(3,4)$.

Rari casi di morte dopo cateterizzazione della vena giugulare interna sono stati segnalati in ampie casistiche e le complicazioni fatali sono state attribuite a sepsi, emotorace, perforazione atriale e a embolia gassosa. È stato anche descritto arresto cardiaco in seguito a palpazione della carotide e a compressione della stessa dopo puntura accidentale dell'arteria.

La vena succlavia viene punta sotto la zona di giunzione tra il terzo medio e la metà della clavicola, previa anestesia locale. La manovra è di relativa, facile esecuzione ed è ben tollerata dal paziente. L'utilizzazione della vena succlavia quale accesso vascolare di emergenza, permette una completa mobilità del malato e una buona utilizzazione dialitica con flussi ematici variabili tra 150 e $250 \mathrm{ml} / \mathrm{m}^{\prime}$. La letteratura segnala elevata incidenza di

\section{TABELLA I - COMPLICAZIONI DEL CATETERISMO DELLA VENA GIUGULARE INTERNA}

$\begin{array}{ll}\text { - Infezioni } & \text { - Trombosi } \\ \text { - Stenosi } & \text { Ematomi } \\ \text { - Malposizionamenti } & \text { - Necrosi cutanee }\end{array}$

TABELLA II - COMPLICANZE DEL CATETERISMO DELLA VENA SUCCLAVIA

- Trombosi della v. succlavia

- Infezioni

- Pneumotorace
- Stenosi della v. succlavia

- Ematomi

- Emotorace complicanze (Tab. II) che talora possono essere di elevata gravità.

La puntura accidentale dell'arteria succlavia è una complicazione relativamente frequente; la pronta estrazione dell'ago e l'applicazione di una accurata compressione digitale sulla sede della puntura cutanea sono per lo più sufficienti a bloccare il sanguinamento e la formazione di ematomi. L'emotorace è una complicanza gravissima, ma relativamente rara, che può comportare pericolo per la vita del paziente. Più frequente è lo pneumotorace che può richiedere il drenaggio d'urgenza e l'assistenza ventilatoria. Sono riportati in letteratura periodi di permanenza in situ del catetere più lunghi rispetto alla via femorale (fino a 60 giorni), ma questo favorisce l'insorgenza di complicazioni tardive (infezione, trombosi, stenosi) (2): tra queste l'infezione è la più frequente e può limitarsi al punto di emergenza della cannula o estendersi al vaso comportando un processo flebitico che può complicarsi con un vero e proprio stato settico.

L'alta incidenza di complicazioni tardive, soprattutto stenotiche, sconsiglia l'uso della vena succlavia come accesso temporaneo per l'emodialisi (5).

La vena femorale viene cannulata nella zona inguinale del triangolo dello Scarpa (dx o sx) con identificazione del punto di introduzione con tecnica manuale (la vena femorale scorre medialmente rispetto all'arteria femorale omolaterale) o mediante Doppler. La manovra di incannulamento della vena femorale viene abitualmente effettuata previa anestesia locale nel rispetto di una scrupolosa asepsi. Nel caso fosse necessaria l'introduzione di due cateteri venosi è opportuno che il loro posizionamento preveda uno sfalsamento dei rispettivi estremi distali di $4-5 \mathrm{~cm}$ sì da ridurre la percentuale di ricircolo che comunque oscilla tra il 10 e il $15 \%$ del flusso.

Il catetere così posizionato può essere lasciato in situ per un periodo di tempo che mediamente oscilla tra i 7 e i 30 giorni: è consigliabile il cambio della cannula ogni 15 giorni. Per la sostituzione si può utilizzare la stessa 

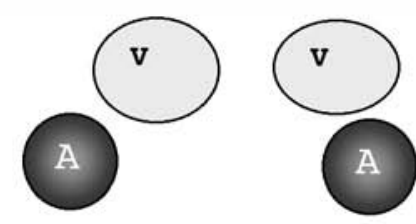

a

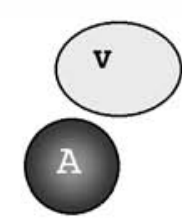

c

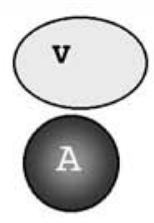

d

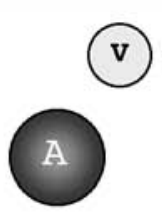

$\mathrm{e}$
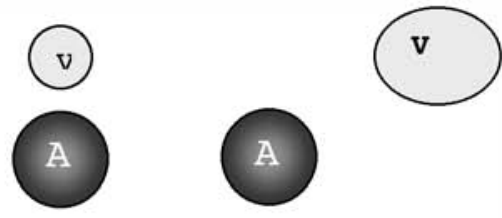

A

f

g

Fig. 1 - Variazioni anatomiche della VGI (da Lin BS. Nephrol Dial Trasplant 1998; 13: 134, modificata).

$V=$ vena giugulare interna $; A=$ arteria carotide .

$a=$ normale $; b=$ invers $a ; c=$ parzialmente sovrapposta $; d=$ sovrapposta $; e=$ piccola normale $;$

$f=$ piccola sovrapposta $; g=$ dislocata .

via in assenza di segni di infezione dell'emergenza cutanea. Le complicazioni del cateterismo della vena femorale possono essere molteplici soprattutto in presenza di fattori di rischio come obesità, edemi, varianti anatomiche dei vasi, ma in genere sono rare e di modesta entità data la mancanza nella sede di organi importanti.

La puntura accidentale dell'arteria femorale è abbastanza frequente, tuttavia la pronta rimozione dell'ago e un'accurata manovra compressiva per 10-15 minuti permettono per lo più di evitare spandimenti emorragici. Talora la formazione di un ematoma può interessare più ampiamente il tessuto sottocutaneo e le fasce muscolari della coscia con temporanea limitazione funzionale dell'arto. Rare complicazioni sono rappresentate dalla trombosi o stenosi della vena femorale e dall'infezione nel punto di introduzione del catetere con conseguente processo flebitico. In tutti questi casi la rimozione del catetere è obbligatoria.

\section{Cannulazione ecoguidata di vena centrale}

È stato segnalato (6) che la localizzazione anatomica manuale per la localizzazione della VGI non è realizzabile in circa il $25 \%$ dei pazienti uremici e ciò prevalentemente per le variazioni anatomiche (Fig. 1) della vena giugulare interna.

Con l'utilizzazione dell'ecografia, la cannulazione ecoguidata di vena centrale è entrata nel bagaglio professionale di molti operatori del settore e ciò ha consentito sia una maggiore sicurezza operativa, sia la significativa riduzione dell'incidenza delle complicazioni acute legate a tale manovra. Dall'analisi della letteratura (Tab. III) risulta infatti che la tecnica ecoguidata comporta sia una drastica riduzione della puntura accidentale dell'arteria, sia una riduzione del numero di tentativi di puntura della vena.

La tecnica ecoguidata real-time prevede l'uso di un trasduttore a $7.5 \mathrm{MHz}$ che, collegato a piccolo ecografo bidimensionale portatile (ad esempio, Site-Rite IV, Dymax Corporation), consente la visualizzazione della VGI data la sua posizione relativamente superficiale e permette di evidenziare le sue variazioni anatomiche nonché la presenza di ostruzione trombotica (Fig. 2).

\section{Modalità operative}

Una volta ottenuto il consenso informato, la manovra di inserzione dell'ago guida viene preceduta da anestesia locale (xilocaina 1\%) nel punto di inserzione dell'ago precedentemente identificato con una semplice scansione ecografica.

A paziente supino la superficie del

TABELLA III - PERCENTUALI DI PUNTURA ACCIDENTALE DI ARTERIA CAROTIDE E DI SUCCESSO DI PUNTURA DELLA VENA. Confronto tra tecnica manuale e tecnica ecoguidata (dati della Letteratura)

\begin{tabular}{|c|c|c|c|c|c|c|}
\hline & $\%$ Successo & $\begin{array}{c}\% \text { Puntura } \\
\text { arteria }\end{array}$ & \% Ematoma & \% Successo & $\begin{array}{c}\% \text { Puntura } \\
\text { arteria }\end{array}$ & \% Ematoma \\
\hline Lin $1998(6)$ & 86 & 4.6 & 7 & 99.0 & 0.9 & 0.9 \\
\hline Farrel 1997 (7) & 82 & 7.7 & $\mathrm{nr}$ & 96.7 & 0.0 & $\mathrm{nr}$ \\
\hline
\end{tabular}




Giornale
di Tecniche
Nefrologiche
\& Dialitiche
Anno XVIII n'4
C) Wichtig Editore 2006
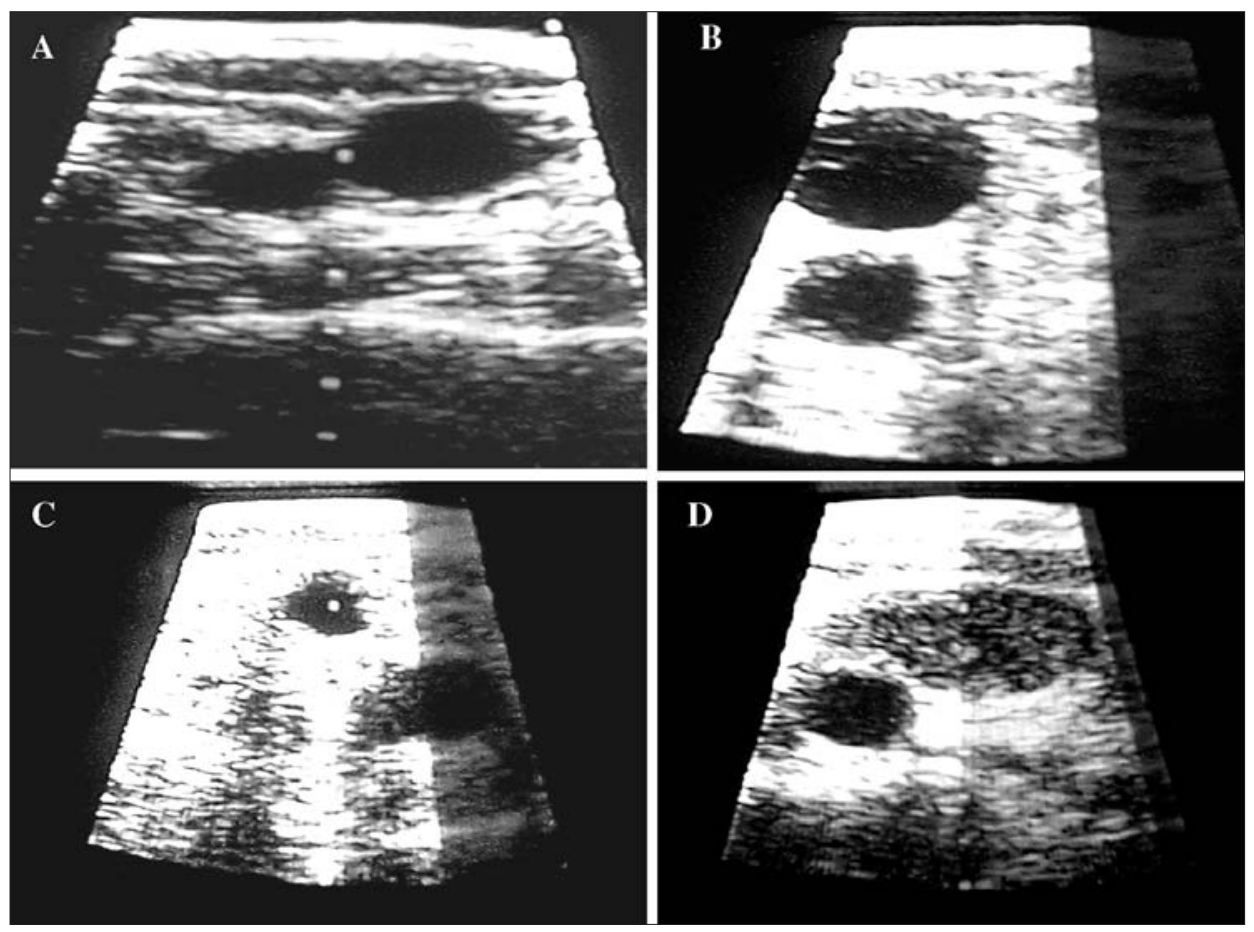

Fig. 2 - Immagini ecografiche della VGI: A = localizzazione normale;

$B=$ localizzazione sovrapposta C $=$ Localizzazione inversa;

$D=$ VGI parzialmente trombizzata .

collo viene opportunamente preparata e disinfettata.

Gel sterile viene applicato sul trasduttore che a sua volta viene coperto da un cappuccio sterile di plastica. Al trasduttore viene infine applicato un supporto sterile di plastica al quale viene agganciato l'ago guida. La cute viene bagnata con soluzione salina sterile e il trasduttore viene posto in corrispondenza del triangolo del Sedillot e più precisamente (ma non necessariamente) nel punto di apparente congiunzione dei due capi lunghi del muscolo sternocleidomastoideo.

Mediante manovra di Valsalva è possibile ottenere un evidente ingrandimento delle dimensioni della vena. La vena può essere facilmente differenziata dall'arteria carotide anche in considerazione del fatto che essa ha solitamente un diametro più ampio, la sua posizione è solitamente in sede laterale e anteriore rispetto all'arteria ed è facilmente compressibile mediante l'applicazione di una moderata pressione.

Usando la tecnica ecografica real time è inoltre possibile la visualizzazione diretta dell'entrata dell'ago guida nella vena. Una volta introdotto l'ago guida nella VGI, la manovra di posizionamento del CVC procede analogamente alla tecnica classica di Seldinger.

\section{CONCLUSIONI}

I dati della letteratura hanno ampiamente dimostrato i vantaggi della cannulazione ecoguidata real-time della VGI rispetto alla inserzione basata sulla localizzazione manuale del punto di inserzione dell'ago (7-11).

Le variazioni anatomiche della vena giugulare interna possono infatti avere un impatto negativo sulla manovra di inserzione del catetere quando viene utilizzata la tecnica tradizionale, e la percentuale di successo di posizionamento di CVC può invece raggiungere il $99-100 \%$ quando si ricorre alla utilizzazione della tecnica ecoguidata. Come più volte segnalato, la cannulazione ecoguidata della vena giugulare interna si associa a significativa riduzione della quota di complicazioni acute e risultati similari sono stati segnalati anche per l'inserzione di catetere nella vena femorale.

In conclusione, si può affermare che la tecnica di cannulazione di vena centrale mediante guida ecografia real-time è di facile uso e gestione, dà sicurezza operativa e consente una significativa riduzione delle complicazioni acute (9-12).

Va infine aggiunto che la tecnica ecoguidata si rivela di particolare utilità nel posizionamento di CVC nella VGI nei pazienti geriatrici quando questi non siano in grado di effettuare la rotazione laterale del capo, sì che la localizzazione manuale del punto di inserzione dell'ago guida diventa particolarmente difficile e a rischio di puntura accidentale di arteria. In tal senso la tecnica ecoguidata real time supera le difficoltà operative di cannulazione di vena centrale secondarie alla ridotta compliance del paziente.

Da queste considerazioni suggeriamo che la cannulazione ecoguidata di vena centrale dovrebbe essere adottata come tecnica abituale per chi pratica tale manovra. 


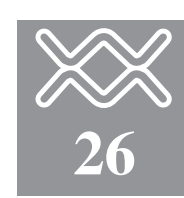

Giornale

di Tecniche

Nefrologiche

\& Dialitiche

Anno XVIII n ${ }^{\circ} 4$

(C) Wichtig Editore 2006

\section{BIBLIOGRAFIA}

1. Seldinger SI. Catheter replacement of the needle in percutaneous arteriography - a new technique. Acta Radiol 1953; 39: 368-76.

2. Cimochowscki GE, et al. Superiority of the internal jugular vein over the subclavian access for temporary dialysis. Nephron 1990; 54: 15461.

3. Fiaccadori E, et al. Cardiac arrhytmias during central venous catheter procedures in acute renal failure: a prospective study. J Am Soc Nephrol 1996; 7: 1079-84.

4. NKF-K/DOKI Clinical Practice guidelines for vascular access. New York, National Kidney Foundation 2001; 28-9.

5. Vanholder R, et al. Morbidity and mortality of central venous catheter hemodialysis: a review of 10 years experience. Nephron 1987; 47: 274-9.

6. Lin BS, et al. Anatomical variation oh the internal jugular vein and its impact on temporary hemodialysis vascular access: an ultrasonographic survey in uraemic patients. Nephrol Dial Trasplant 1998; 13:134-8.

7. Farrel J, et al. Ultrasound guided cannulation versus landmark guide technique for acute hemodialysis access. Nephrol Dial Trasplant 1997; 12: 1234-7.

8. Denys BG, et al. Ultrasound assisted cannulation of the internal jugular vein: a prospective comparison to the external guided technique. Circulation 1993; 87: 1557-62.

9. Conz PA. Cannulation of the internal jugular vein: comparison of the classic Seldinger technique and an ultrasound guide method. J Nephrol 1997; 87: 1557.

10. Hatfield A, et al. Portable ultrasound for difficult central venous access. Br J Anaesth 1999; 6: 822-6.

11. Aslam N, et al. Real time ultrasound for placement of dialysis ca- theter: a new standard of care. Semin Dial 1999; 1: 1-4.

12. Conz PA. Slow maturation of arterio-venous fistula in seven uremic patients: use of Ash Split cath as temporary, prolonged vascular access. J Vasc Access 2000; 1: 51-3. 